\title{
Value of CT scanning in the diagnosis and management of patients admitted with stroke to a tertiary care center in Sri Lanka
}

\author{
GJ Pratheepan ${ }^{1}$, DBYN de Silva ${ }^{2}$, TP Weerarathna ${ }^{3}$ \\ ${ }^{1}$ Registrar in Medicine, ${ }^{2}$ Senior Registrar in Medicine, ${ }^{3}$ Professor in Medicine, University Medical Unit, \\ Teaching Hospital, Galle.
}

\begin{abstract}
Introduction: Stroke is a clinical diagnosis for which CT is the mainstay of investigation. The diagnostic comparisons between the clinical and the CT diagnosis of stroke and the impact of the latter on management are unclear in the local setting.

Materials and Methods: Fifty admissions with the clinical diagnosis of stroke to the University Medical unit were studied for three months. Data on the time and mode of admission, the sub-type of clinical diagnosis (infarct, intracerebral and subarachnoid haemorrhage), CT diagnosis and the impact of CT diagnosis on the management were recorded.

Results: Females accounted for 52\%. Cerebral infarctions were commoner than the haemorrhage (56\% and $12 \%$, respectively). The sub-typing of stroke was identical to the clinical diagnosis in $44 \%$ of patients. The sensitivity of the clinical diagnosis is highest in SAH (75\%) followed by infarction (61\%) and ICH (6\%). The specificity was highest in SAH (98\%) followed by ICH (70\%) and cerebral infarction $(36 \%)$.

Conclusion: CT brain has improved the diagnostic accuracy of stroke. It has contributed for better management. As the clinical diagnosis of the sub-typing of stroke is correct in less than 50\%, CT scan is mandatory for all patients with stroke in the local setting.
\end{abstract}

\section{Introduction}

With the rising burden of non-communicable disease and aging population in the community, morbidity and mortality due to stroke have shown an increasing trend worldwide ${ }^{1}$. In Sri Lanka, it is the $5^{\text {th }}$ leading cause of death among people admitted to public sector hospitals ${ }^{2}$.

In 2005, the annual incidence of deaths due to stroke was 2549 . Proportionate mortality was $7.7 \%$ and the rate per 100000 was 13.1.

Stroke is defined as a clinical syndrome of rapid onset of focal or global cerebral deficit lasting for more than 24 hours or leading to death. Aetiologically, $80 \%$ of strokes occur due to cerebral infarcts and intracerebral haemorrhages $(15 \%)$ and subarachnoid haemorrhages $(5 \%)$ account for the remaining $20 \%{ }^{3}$.

Severe headache, convulsions, neck stiffness and progressive deterioration of neurological deficit from the onset are known to favour underlying cerebral haemorrhage. In contrast, absence of headache and neck stiffness, maximal neurological deficit at the onset favours cerebral infarct ${ }^{4}$. However, these clinical features vary in sensitivity and specificity in a given setting.

With the discovery of benefits of modern therapeutic interventions for sub-types of stroke (thrombolytic and anti-platelet therapy ${ }^{5}$ for cerebral infarcts, and optimal blood pressure lowering and specific agents like Nimodipine for subarachnoid haemorrhage), early differentiation of the underlying pathology of stroke can become a crucial issue in the therapeutic decision making in a given patient.

Advent of cerebral imaging methods such as Computerized Tomography (CT) has enabled early and precise differentiation of cerebral infarct from haemorrhage ${ }^{6}$.

Information thus obtained is useful in therapeutic decision making such as administration of thrombolytics (where it is available) and early use of anti-platelet therapy for the category of patients with cerebral infracts. Delays in seeking medical attention or 
poor access to computed tomography for stroke will result in failure to identify up to three quarters of intracerebral haemorrhage and may lead to inappropriate treatment ${ }^{7}$.

CT scanning facility was installed in the Teaching Hospital Galle in 1996. Now it is a routine practice to order a $\mathrm{CT}$ scan for subjects with a clinical diagnosis of stroke admitted to this Institute. The radiology department of the Hospital carries out an average of $580 \mathrm{CT}$ scans per month, out of which 480 are brain scans, with an average of 16 brain scans per day.

The Value of CT scanning in the differential diagnosis and management of patients with stroke has not been evaluated in the local setting. This information would be useful in selecting the most suitable patients for CT scan and using the facility cost effectively in the limited resource setting.

\section{Objectives}

1. To compare the clinical diagnosis of the sub-type of stroke with the CT diagnosis.

2. To determine the impact of the CT diagnosis on the therapeutic decision making and early management of stroke.

\section{Methods}

This study was carried out in one of the five medical units in Teaching Hospital, Galle. Fifty consecutive admissions with clinical diagnosis of stroke to the University Medical Unit were studied. Data on the demographic profile, subtype (infarct or haemorrhage) of stroke on clinical grounds and subsequent therapeutic interventions including use of antiplatelet agents, neurosurgical referral and/or intervention were entered on to a pre-designed data entry form.

Clinical diagnosis of intracranial haemorrhage was made in the presence of loss of consciousness and progressive deterioration of neurological deficit from the onset of symptoms with or without convulsions. Diagnosis of subarachnoid haemorrhage was clinically made in the presence of sudden, severe headache and neck stiffness. Absence of above findings with sudden onset of focal or global neurological deficit which was maximal at the onset of symptoms was clinically categorized as cerebral infarction

All patients underwent CT scanning of brain within twenty four hours of admission to the Unit.

\section{Results}

The total number of patients admitted to the Unit during the study period was 3002 with 1616 males. Admissions with stroke accounted for $1.7 \%$ of total admissions. Out of 50 admissions $48 \%$ were males and $52 \%$ were females. Direct admissions accounted for a majority (72\%). Internal transfers and transfers from other hospitals accounted for $6 \%$ and $22 \%$, respectively.

The mean age of subjects with stroke was 70 years (68 years for males and 72 years for females, respectively) (Table 1). Thirty eight out of 50 (i.e. $76 \%$ ) of patients were admitted within 24 hours of onset of symptoms. Based on clinical features, $62 \%$ of patients were categorized as cerebral infarcts and $30 \%$ and $8 \%$ were diagnosed as intracerebral and subarachnoid haemorrhage, respectively (Fig 1). CT scanning revealed that $8 \%$ of patients with clinical diagnosis of stroke were having other cerebral pathology (4\% hydrocephalus, $4 \%$ cerebral tumor) and $16 \%$ of patients with a clinical diagnosis of stroke had a normal CT.

Overall, clinical diagnosis of pathological subtype of stroke was identical to the CT diagnosis in 22 cases (44\%) (Table 2). Eight percent of the clinically suspected cerebral infarcts were found to be haemorrhages and $20 \%$ of clinically suspected haemorrhages were found to be cerebral infarcts on the CT.

Out of ten cerebral haemorrhages confirmed on CT, nine were referred for neurosurgical opinion, but none underwent neurosurgical intervention. Antiplatelet therapy with low dose aspirin and dipyridamole was commenced soon after the exclusion of cerebral haemorrhage in $64 \%$. Twelve subjects $(24 \%)$ who would have been deprived of antiplatelet therapy on clinical diagnosis of stroke were benefited by performing a CT scan. Four subjects $(8 \%)$ who could have been given antiplatelet therapy on 
clinical grounds were prevented from receiving antiplatelet therapy as the CT scan has revealed $\mathrm{ICH}$ in them.

Table1 - Characteristics of study sample and symptom profile

\begin{tabular}{|lll|}
\hline Male : female & $1: 1.08$ \\
Age & $\begin{array}{l}70.02 \text { Year } \\
\text { (SD 12.29) }\end{array}$ \\
\% of admission < 24 hours & $76 \%$ \\
\% of admission > 24 hours & $24 \%$ & \\
Mode of admission & & \\
$\quad$ Direct & $72 \%$ & \\
Transfer & $26 \%$ & \\
Referral & $06 \%$ & \\
Symptom profile & & \\
Headache & 12 & 24 \\
Loss of consciousness & 15 & 30 \\
Hemi paresis & 27 & 54 \\
Monoparesis & 05 & 10 \\
Aphasia & 10 & 20 \\
Dysphasia & 04 & 08 \\
\hline
\end{tabular}

Table2 - Clinical and CT diagnosis

\begin{tabular}{|l|c|c|c|c|}
\hline \multirow{2}{*}{$\begin{array}{c}\text { CT } \\
\text { diagnosis }\end{array}$} & \multicolumn{4}{|c|}{ Clinical diagnosis } \\
\cline { 2 - 5 } & Infarction & ICH & SAH & Total \\
\hline Infarction & 17 & 10 & 01 & 28 \\
\hline ICH & 04 & 02 & 00 & 06 \\
\hline SAH & 00 & 01 & 03 & 04 \\
\hline $\begin{array}{l}\text { Normal } \\
\text { CT }\end{array}$ & 07 & 01 & 00 & 08 \\
\hline Others & 03 & 01 & 00 & 04 \\
\hline Total & 31 & 15 & 04 & 50 \\
\hline
\end{tabular}

Table 3 - Sensitivity and specificity of clinical diagnosis

\begin{tabular}{|l|c|c|c|c|}
\hline $\begin{array}{c}\text { Clinical } \\
\text { diagnosis }\end{array}$ & Sensitivity & Specificity & PPV & NPV \\
\hline Infarction & 60.71 & 36.36 & 0.55 & 0.42 \\
\hline ICH & 06.06 & 70.45 & 0.13 & 0.88 \\
\hline SAH & 75.00 & 97.82 & 0.75 & 0.98 \\
\hline
\end{tabular}

$P P V$ - positive predictive value,

$N P V-n e g a t i v e$ predictive value

Figure 1 - Clinical diagnosis

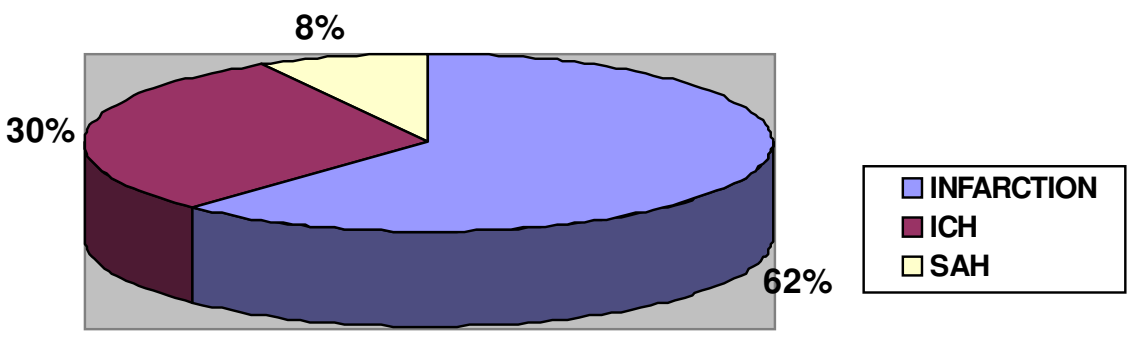

Figure 2 - Frequency distribution of symptom profile

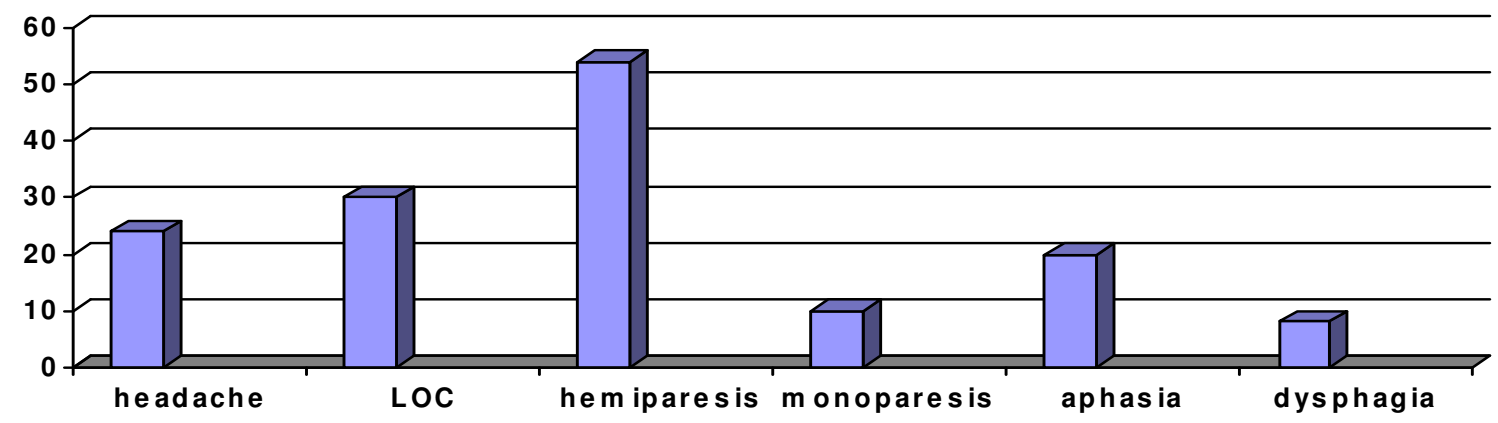


Figure 3 - Sensitivity, specificity, positive and negative predictive values of clinical diagnosis

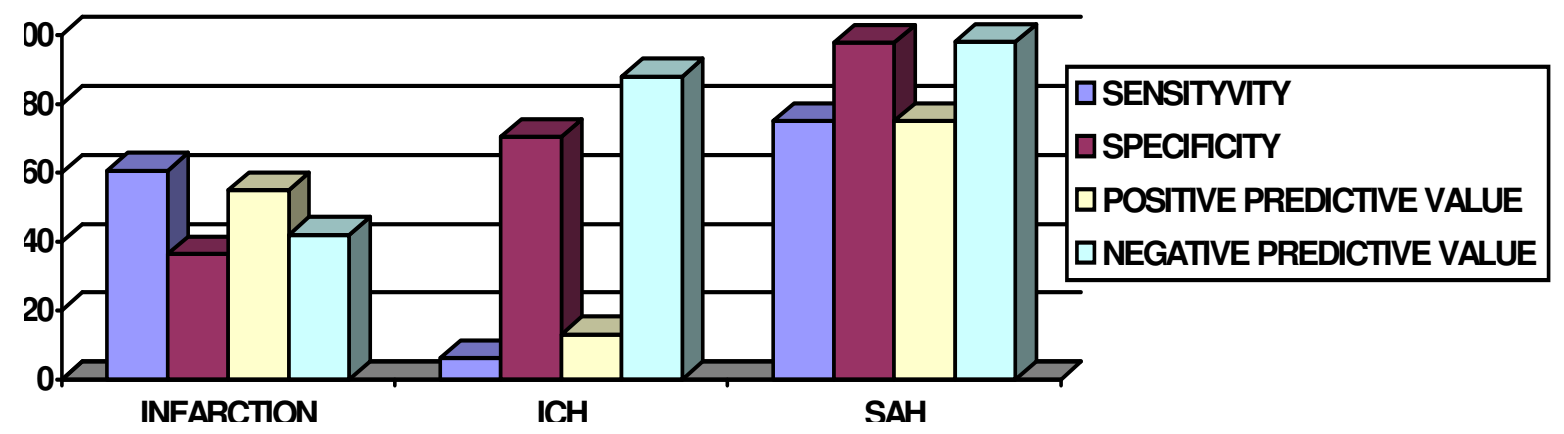

\section{Discussion}

Introduction of evidence based treatment modalities for patients with acute stroke has compelled clinicians to arrive at a precise diagnosis of the category of stroke and thereby to initiate optimal management. Although Modern neuroimaging has enabled early diagnosis of the event, limited availability and high cost of investigations have restricted their use only in tertiary care centers ${ }^{8}$. Therefore, clinical diagnosis is still relied upon in primary care settings.

This study was designed to compare the accuracy of clinical diagnosis of stroke with CT scanning as the gold standard. It also evaluated the impact of CT diagnosis in the initial management.

The main finding of this study was that the clinical diagnosis of stroke and its sub-types were identical with the CT diagnosis only in $44 \%$ of patients. Clinical diagnosis of infarction was more precise than that of cerebral haemorrhage.

SAH had the highest sensitivity and specificity (75\%) and (98\%), respectively. Infarctions had a moderate amount of sensitivity $(61 \%)$ but low level of specificity (36\%) ICH had lowest level of sensitivity $(07 \%)$ but moderate amount of specificity (70\%) (Fig 3). This points out the need for CT scanning in all patients with stroke ${ }^{9}$.

However, there is a possibility that infarctions might have been missed because of the early CT scans ${ }^{10}$ and the routine slicing with $10 \mathrm{~mm}$ cuts. Cerebral haemorrhages could also have been missed due to the same slicing difference as well as due to the low sensitivity ${ }^{11}$ of CT scans over MRI scans in detecting haemorrhages. However, all patients in whom the CT has been negative for a cerebral haemorrhage were treated as subjects with cerebral infarctions although there is an existing remote possibility of having a cerebral haemorrhage. Performing an early CT has helped in confirming or excluding cerebral haemorrhage thereby helped in early administration of antiplatelet therapy which would be a therapeutic dilemma otherwise. Optimizing the blood pressure up to the therapeutic targets according to the stroke subtypes and appropriate neurosurgical referral were also achieved by doing an early CT scan.

Superiority of neuroimaging over the bedside diagnosis of stroke has already been revealed in previous studies ${ }^{12}$ which utilized standard scoring systems for the clinical diagnoses.

It was also shown that in the local setting, by categorization of the sub-types of stroke by the CT scan prevented adverse management strategies.

Twelve subjects (24\%) who would have been deprived of antiplatelet therapy on clinical diagnosis of stroke were benefited by performing a CT scan. Four subjects (8\%) who could have been given antiplatelet therapy on clinical grounds were prevented from antiplatelet therapy as the CT scan has revealed $\mathrm{ICH}$ in these patients. Thus, the overall beneficiaries were $32 \%$. 
Neurosurgical intervention was not carried out in any of the nine subjects who had intracerebral haemorrhage. This could have been due the nature of the haemorrhage or the clinical condition of the patient.

In this era of thrombolysis for acute ischemic infarctions, early and accurate diagnosis ${ }^{13}$ of such events should be available for the optimal patient care.

In this regard, it is encouraging that the majority of patients in this study sought medical attention from this tertiary care centre by themselves or through a referral system within 24 hours. Even though patients seeked early medical attention following a stroke, providing appropriate thrombolysis is still out of scope of this institution. The National hospital of Sri Lanka is the only institute which is able to provide this service to selected patients at the moment. Further investigating the cause and the nature of the stroke sub-types by other imaging modalities have not been the practice, mainly due to the limited resource setting and the non availability of some imaging modalities such as perfusion scans.

Several limitations were inherent to this study. The criteria for the clinical diagnosis were not based on a standard scoring system. The longterm benefits of early initiation of antiplatelet therapy have not been evaluated as it was out of scope of the objectives of this study. As the study sample was small in some of the sub-types, the statistical significance of the results of such categories could be questionable.

\section{Conclusions}

Availability of CT scanning facility has refined the diagnostic accuracy of stroke in the local setting. It has also contributed for the better management of stroke during the acute stage. As the clinical diagnosis of the sub-typing of stroke is correct in less than $50 \%$ of instances, CT scan should be a mandatory investigation for all patients with the clinical diagnosis of stroke in the local setting.

\section{Recommendations}

We recommend that all the patients with the clinical diagnosis of stroke should be referred early to a centre where CT scanning is available. A standard scoring system should be adhered to when arriving at a clinical diagnosis of stroke. Improvements in the care of patients with stroke such as dedicated stroke units with facilities for thrombolysis should be available.

\section{Acknowledgements}

We thank the medical staff of the University Medical Unit for the assistance in data collection, Dr. (Mrs.) Padmini Kolombage and Dr. A.F. Fathihu, Consultant Radiologists of this hospital for reporting CT images, Professor Sarath Lekamwasam for advice and help during the statistical analysis.

\section{References}

1. Danaei G, Lawes CM, Vander Hoorn S, Murray CJ, Ezzati M. Global and regional mortality from ischemic heart disease and stroke attributable to higher-thanoptimum blood glucose concentration: comparative risk assessment. Lancet. 2006 Nov 11; 368(9548): 1651-9.

2. Leading causes of hospital deaths, Morbidity and mortality, Annual health bulletin - Sri Lanka 2005

3. Hatano S. Experience from a multicentre stroke register: a preliminary report. Bull WHO 1976; 54: 541-53.

4. CRA Clarke. Neurological disease. In: Kumar \& Clerk. Clinical Medicine, $6^{\text {th }}$ edition, Elsevier Saunders, Edinburgh, 2005: 1173-271.

5. Chen ZM, Sandercock P, Pan HC, Counsell C, Collins $\mathrm{R}$, Liu LS, et al. Indications for early aspirin use in acute ischemic stroke: A combined analysis of 40000 randomized patients from the Chinese acute stroke trial and the international stroke trial. On behalf of the CAST and IST collaborative groups. Stroke 2000; 31: 1240-9.

6. Keir SL, Wardlaw JM, Sandercock PA, Chen Z. Anti thrombotic therapy in patients with any form of intracranial haemorrhage: a systematic review of the available controlled studies. Cerebrovascular Disease 2002; 14: 197-206.

7. Wardlaw JM, Keir SL, Dennis MS. The impact of delays in computed tomography of the brain on the accuracy of diagnosis and subsequent management in patients with minor stroke. Journal of Neurology, Neurosurgery and Psychiatry 2003; 74: 77-81. 
8. Ogun SA, Oluwole O, Fatade B, Ogunseyinde AO, Ojini FI, Odusote KA. Comparison of Siriraj Stroke Score and the WHO criteria in the clinical classification of stroke sub-types. African Journal of Medical Sciences 2002 Mar; 31(1): 13-6.

9. Hawkins GC, Bonita R, Broad JB, Anderson NE. Inadequacy of clinical scoring systems to differentiate stroke sub-types in population-based studies. Stroke. 1995 Aug; 26(8): 1338-42.

10. Fiebach JB, Schellinger PD, Jansen $\mathrm{O}$, Meyer $\mathrm{M}$, Wilde P, Bender J, et al. CT and diffusion-weighted MR imaging in randomized order: diffusion-weighted imaging results in higher accuracy and lower interrater variability in the diagnosis of hyperacute ischaemic stroke. Stroke 2002; 33: 2206-10.
11. J Wardlaw, S Keir, and M Dennis. The impact of delays in computed tomography of the brain on the accuracy of diagnosis and subsequent management in patients with minor stroke, Journal of Neurology Neurosurgery and Psychiatry 2003 January; 74(1): 7781.

12. Ozeren A, Bicakci S, Burgut R, Sarica Y, Bozdemir $\mathrm{H}$. Accuracy of bedside diagnosis versus Allen and Siriraj stroke scores in Turkish patients. European Journal of Neurology 2006 Jun; 13(6): 611-5.

13. Fiebach JB, Schellinger PD, Gass A, et al. Stroke magnetic resonance imaging is accurate in hyperacute intracerebral haemorrhage: a multi center study on the validity of stroke imaging. Stroke 2004 Feb; 35(2): 502-6. 